\title{
System for the Dielectric Evaluation of Powders and Liquids
}

\author{
Youssef El gholb, Yaqiang Liu and Paul O'Leary \\ Engineering Department, Waterford Institute of Technology, Waterford, Ireland . \\ Email: yelgholb@wit.ie
}

\begin{abstract}
A$ free-space broadband measurement technique is proposed to determine the dielectrics properties of powders and liquids. Two techniques are presented based on horizontal and vertical propagation, both of which allow for a series of measurements to be taken where the varying parameter is thickness of material under test (to overcome the challenge of the unknown electrical length). The horizontal propagation measurements required an extra containment layer, which had consequent mechanical and also computational difficulties. The vertical propagation measurements on the other hand show more promise, both in terms of mechanical containment and also in the inversion of the measured S-parameters to arrive at the complex electric permittivity. The measurements were performed in a large hall and also in an anechoic chamber. To minimise measurement errors a free-space $\underline{T}$ hru- $\underline{R}$ eflect-Line/Match (TRL/M) calibration technique was performed. The initial results reported here are for various glass thicknesses in the frequency range of 0.8-6 GHz, only although the application to powders and liquids will be apparent.
\end{abstract}

Index Terms - TRL/M Calibration, Non-Destructive Testing measurement, Vector Network Analyser, Dielectric Materials, Complex Permittivity

\section{INTRODUCTION}

Wireless signals can be used to obtain a deeper understanding of a material's nature through Non-Destructive Testing (NDT). The change in a signal's parameters because it passed through a Material Under Test (MUT) can be used to identify the material's properties, for example, if the material is to be heated in microwave ovens (such as food, adhesives, wood, plastics, etc.), or if communication signals are expected to pass through the material (for example, walls, windows, etc.).

A dielectric measurement technique has been developed which can be used to measure both solid and non-solid dielectrics. Free space non-destructive testing of material dielectric properties encounters a challenge when the electrical length of the material is unknown. Conventional techniques are based on estimates of the electrical length and then an iterative process to refine the estimate. This work presents an alternative to the conventional techniques, by taking measurements over several material thicknesses. This leads immediately to a solution for the material wavelength and thereafter the material dielectric response. The proposed technique should work well for dielectric powders, liquids and gases and requires a modification to a previously developed dielectric analysis structure [3], to permit thickness variations of the material under test. This is a broadband, free-space technique, which has advantages over alternative more frequency- and sample-limited methods using microwave probes or waveguides.
The propagating microwaves will change due to the presence of the dielectric material and this can be used to extract the material's permittivity (and permeability) values. This calculation starts by measuring the material's Sparameters by using VNA over a wide range of frequencies (here 800 to $6000 \mathrm{MHz}$ )

\section{MEASUREMEnt TeChNiQUE AND Framework}

Both the horizontal and vertical techniques presented here measure the material at various thicknesses, to overcome the uncertainty relating to electrical length. This is presented in simple form in Figure 1.

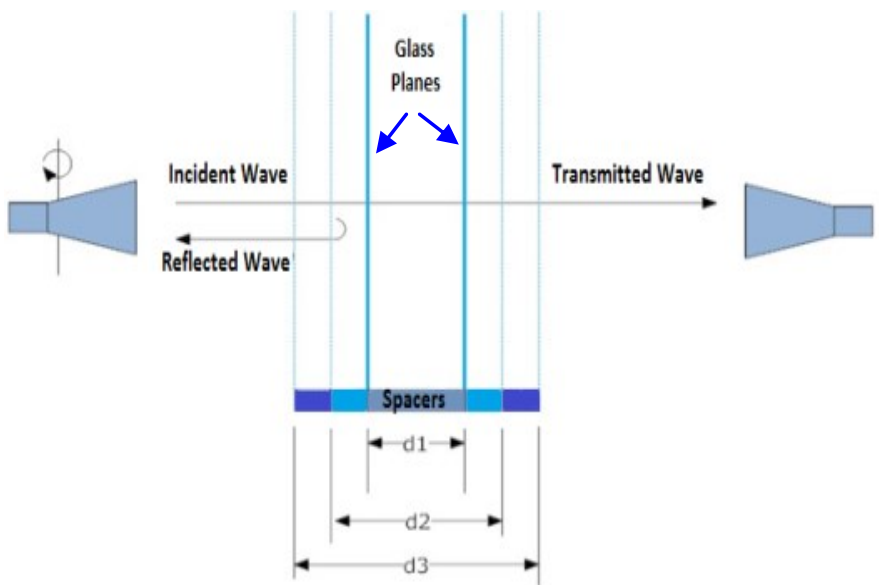

Fig. 1 Antennas mounted on a modified Pentax Precision Total Station

The reduction of errors in the measurement system was effected using the TRL/M method, shown in block diagram form in Figure 2, for free-space and is fairly easily achieved with this test platform.

In this case, TRL/M stands for,

- Thru: Antennas facing each other normally

- Reflect: Metal reflector same size and placed in the same position as the Material Under Test

- Line: Antennas moved by up to $\pm \lambda / 4$ longer than Thru, at centre frequency, to avoid a singularity. Ratio of start-stop frequencies is less than 8:1

- Match: Absorbing foam at the same position as the reflector 


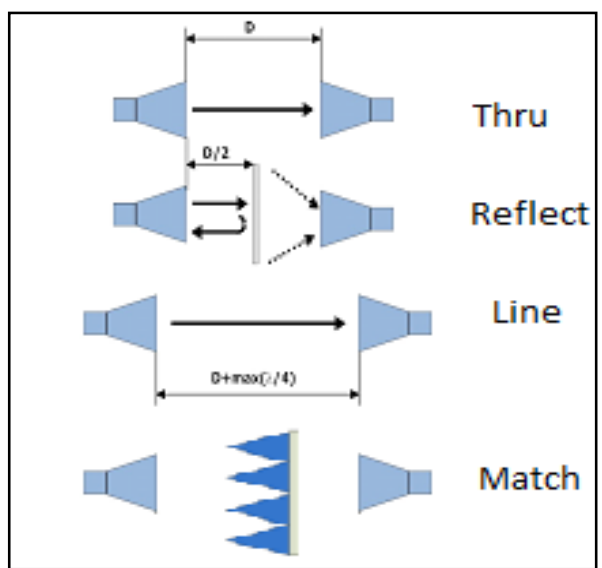

Fig. 2: TRL/M Calibration

\section{A. Horizontal Measurement Method}

Free-space broadband dielectric measurement of powders and liquids presents particular problems that originate with powder/liquid containment to present a uniform and homogenous profile to the probing electromagnetic waves. The form the containment takes also impacts the inversion challenge from measured S-parameters to complex permittivity results.

As the originally developed test framework [3] used horizontally propagating waves, this approach was also taken initially when considering the challenge of measuring powders and liquids.

The propagation properties were measured using Schwarzbeck BBHA 9120 horn antennas. The antennas were mounted on a modified Pentax Precision Total Station [7] (Figure 3), which permitted accurate distance and angular measurements. The measurements were performed with a Rohde \& Schwarz ZVB-20 Vector Network Analyser (VNA). The Rhode \& Schwarz calibration kit ZV-Z32 was used for the cable calibrations. The cables chosen were $7.62 \mathrm{~m}$ long Sucoflex 106, low-loss cables from Huber and Suhner. A glass tank is developed for non-solid material test, with a variable thickness.

Horizontal measurements however, require a verticallyorientated material and in the case of free-flowing materials such as liquids and powders, this means a container is required, especially as material homogeneity and thickness is required. The challenge is exacerbated here with the need to take measurements at various material thicknesses.
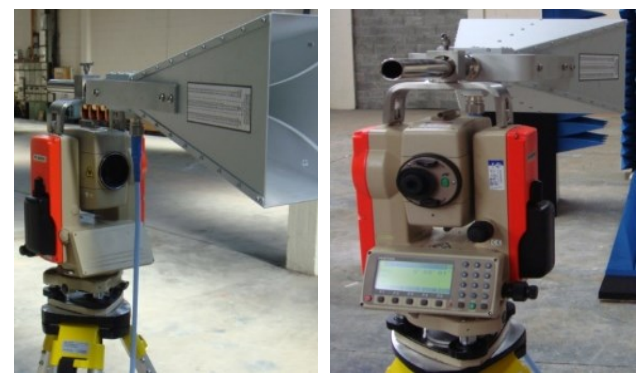

Fig. 3: Antennas mounted on a modified Pentax Precision Total Station

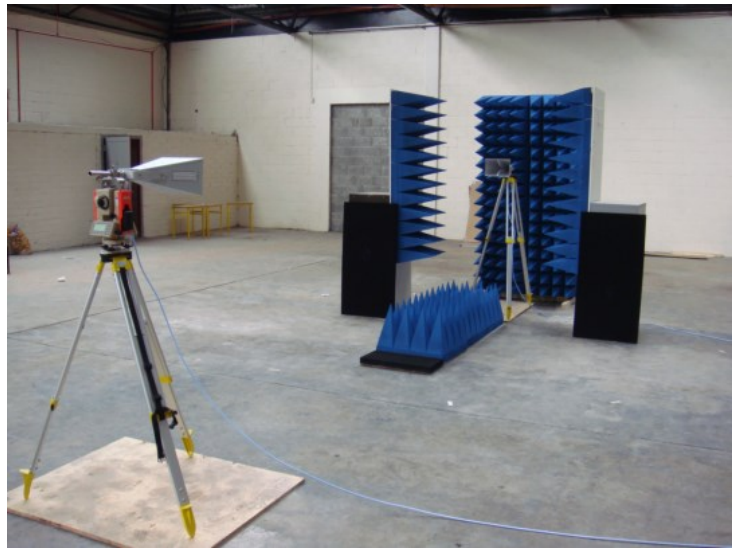

Fig. 4: Horizontal large hall measurements

Requirements of the containment structure include therefore that it should offer flexible, but consistent depth, and offer a wide target to improve accuracy. Ideally the containment walls should therefore be rigid, unyielding under the pressure of the MUT. The depth variability and width requirements implies a large volume of material may be required for the larger thicknesses (up to $200 \mathrm{~mm}$ is possible with the current set-up, while the width and height are $40 \mathrm{~cm}$ and $30 \mathrm{~cm}$ respectively. Various thicknesses of standard glass was used initially for the container walls, even though its permittivity presented an inversion challenge in the dielectric calculations. It offered the advantage, however, of visible examination of material distribution (in the case of powders). It was later replaced with an opaque plastic whose permittivity constant was approximately 1 over the measurement band.

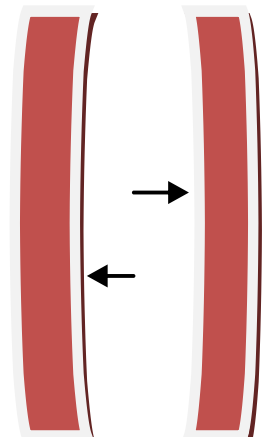

It became obvious early on in the development of the horizontal test structure that the combined rigidity and flexible depth requirements were very difficult to meet. Perfect rigidity was difficult, even with $12 \mathrm{~mm}$ thick glass. Values lower than this thickness exhibited a lens effect, shown in block diagram form in Figure 5 and in a measurement of table salt in Figure 6. One consequence of this is surprisingly large values of $S_{21}$ values at some frequencies (see Figure 8), even when the lens effect is not so obvious to the human eye. 


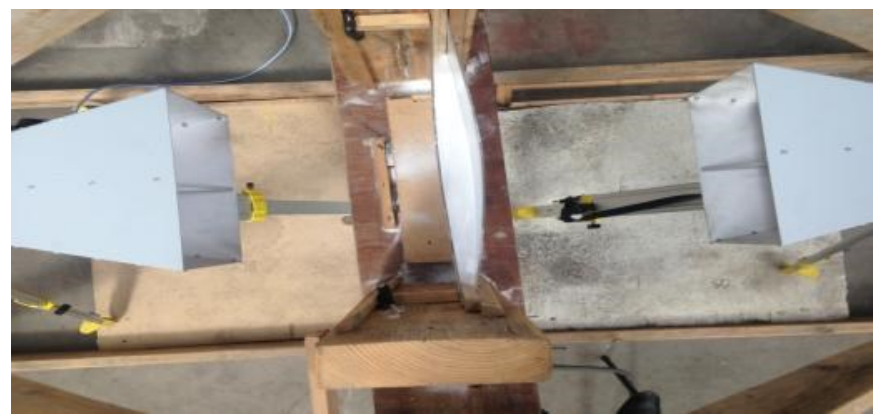

Fig. 6: Lens effect in salt test (centre of picture)

The requirements to measure materials at several thicknesses (depth flexibility in the container) also presented challenges of misalignment of container walls. These produced apparently anomalous results until deliberate misalignment reconstructions (Figure 7) were measured to confirm this as the source of the anomalies.

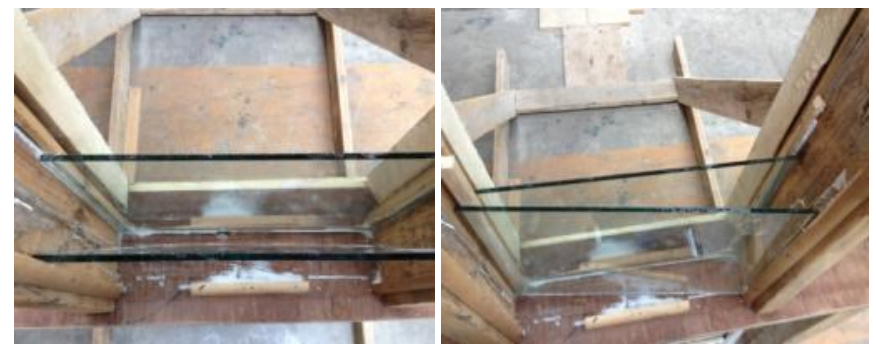

Fig. 7: Misalignment reconstruction tests

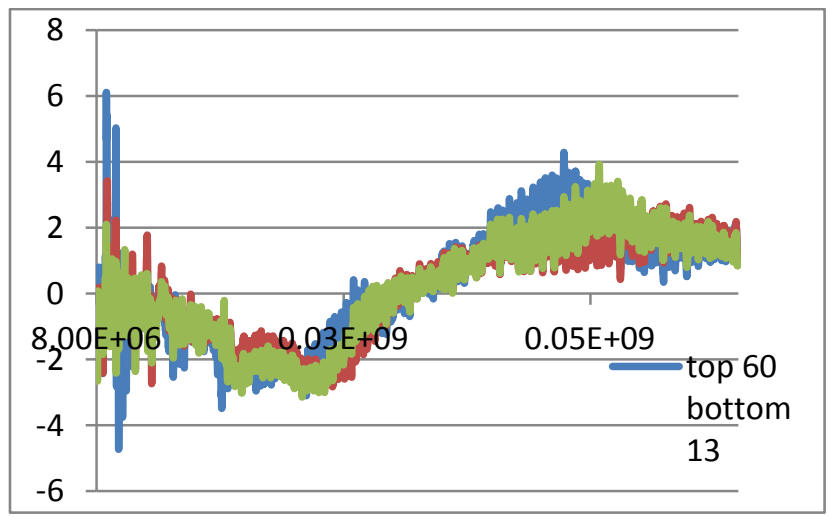

Fig. 8: Typical misalignment measurements

\section{B. Vertical Measurement Method}

Although a vertically aligned test infrastructure presents particular structural challenges in particular in stability, normal incidence and alignment, some benefits are immediately apparent compared with the horizontal arrangement. Immediately the containment requirement is simpler and the flexible depth to allow variable MUT thickness is also straightforward. Figure 11 illustrates a proposed structure to implement a vertically aligned measurement in an anechoic chamber. This structure consists of one layer of glass beneath the material instead of two in the horizontal structure, which also allows an easier inversion (no $\mathrm{ABCD}$ matrix requirements) in the permittivity calculations. Once fixed in place, it also permits normal incidence of the waves with more accuracy. It is excellent for high permittivity values (short electrical length, as the MUT can easily be a depth of $5 \mathrm{~mm}$ or less. It also provides simple and easy measurements of MUT thickness variation (powders) and is particularly easy to test liquids.

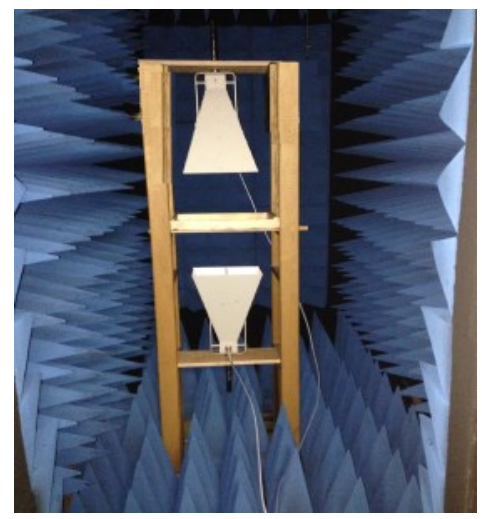

Fig. 9: Candidate vertical measurement system

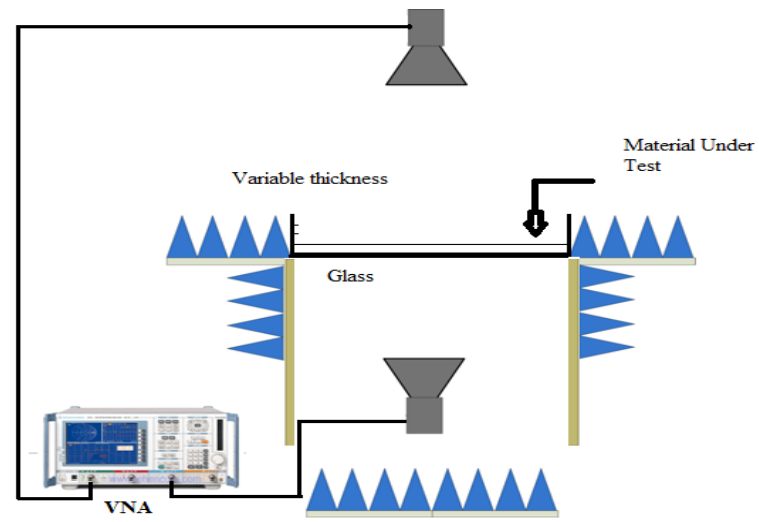

Fig. 10: Block diagram of vertical measurement system

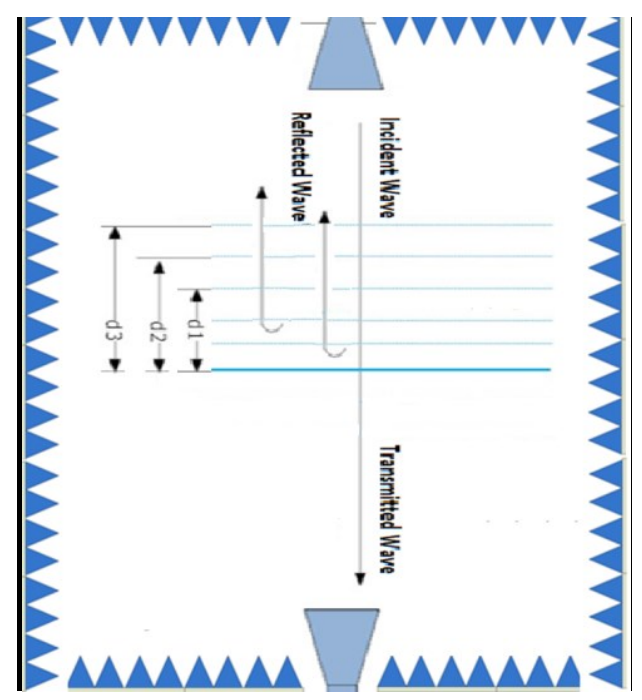

Fig. 11: Vertical anechoic chamber measurements 


\section{Inversion Algorithm}

The calibrated scattering parameters, $\mathrm{S}_{21}$ and $\mathrm{S}_{11}$ are easily obtained using a VNA. The reflection and transmission coefficients are related to the scattering parameters:

$$
\begin{aligned}
& S_{11}=\frac{\Gamma\left(1-T^{2}\right)}{1-I^{2} T^{2}} \\
& S_{21}=\frac{T\left(1-I^{2}\right)}{1-T^{2} \Gamma^{2}}
\end{aligned}
$$

Which can be inverted, so that the reflection coefficient, $\Gamma$, is and transmission coefficient, $\mathrm{T}$, can be related to the permittivity, the permeability and the propagation constant by:

$$
\begin{gathered}
\varepsilon=\frac{\gamma}{\gamma_{0}}\left(\frac{1-\Gamma}{1+\Gamma}\right) \\
\mu=\frac{\gamma}{\gamma_{0}}\left(\frac{1+\Gamma}{1-\Gamma}\right) \\
\gamma=\left[\log _{e}(1 /|T|)\right] / d+j\left(\frac{2 \pi n+\phi}{d}\right)
\end{gathered}
$$
$2, \ldots$.

where $d$ is the thickness of material under test and $n=0,1$,

The real part of the propagation constant is single valued, but the imaginary part may have multiple values. So, imaginary part of is the phase constant

$$
\begin{gathered}
\beta=\frac{2 \pi}{\lambda m} \\
\frac{d}{\lambda_{m}}=n+\frac{\phi}{2 \pi}
\end{gathered}
$$

- When $\mathrm{n}=0$ and $0<\phi<2 \pi, \mathrm{d} / \lambda \mathrm{m}$ is between 0 and 1 . If the thickness $d$ is less than $\lambda \mathrm{m}$, there will be a unique value for the complex permittivity and permeability.

- When $\mathrm{d}>\lambda \mathrm{m}$, one solution is to make the measurements for at least two different thicknesses of the material under tests as first proposed in [4] and explained in more detail below.

Equation (7) is the key to solving for propagation in a MUT. However, solving the imaginary part presents a challenge, unless the material width, $d$, happens to be smaller than the material wavelength, $\lambda_{m}$. This is difficult to guarantee, even for low values of permittivity, over the range of frequencies tested so far, it is then impossible to guarantee this condition.

For any material thickness, $d_{i}$, the measured phase change, $\phi_{i}$, and the material wavelength, $\lambda_{m}$, are related by:

$$
d_{i}=n_{i} \lambda_{m}+\frac{\phi_{i}}{2 \pi} \lambda_{m}, 0<\phi \mathrm{i}<2 \pi
$$

The material thickness, $d_{i}$, is known and the phase change, $\phi_{i}$, can be measured, but neither the material wavelength, $\lambda_{m}$, nor the number of wave periods in the material, $n_{i}$, are known. Both are required in the solution of (8).

The control of the variation in the thickness $d_{i}$, when measuring the dielectric response of the material under test will allow the experimenter to avoid a situation where the new thickness is an integer multiple of material wavelengths more than the previous thickness. Clearly this will then yield two linear equations, where the thicknesses and phase changes can be measured, leading to an easy solution for the material wavelength, $\lambda_{m}$, and the number of wave periods in the material, $n_{i}$.

\section{RESULTS}

This is relatively early in the testing stage of the system, so the first results presented are for solid glass at thicknesses of 4, $6,8,12$ and $15 \mathrm{~mm}$. Glass was chosen due to its availability, previously published data for benchmarking, possible use for containment purposes in the final system, rigidity and ease of material inspection at the early stages of development. Furthermore, the permittivity of glass will be calibrated out in the vertical system, as only a single containment (i.e. not a sandwich) layer is required.

The results presented here were measured using the horizontal system $(6 \mathrm{~mm}$ and $12 \mathrm{~mm})$ in a large hall, as well as the vertical system $(4 \mathrm{~mm}, 8 \mathrm{~mm}$ and $15 \mathrm{~mm})$ in an anechoic chamber. The results show reasonable agreement with each other and with the published values of permittivity constant for glass (3-15 dielectric constant figures).

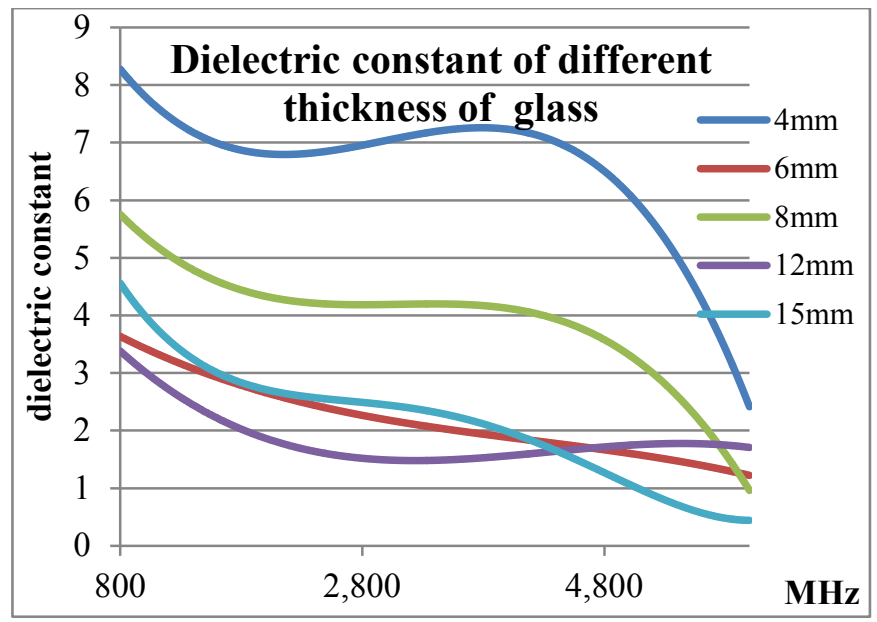

Fig. 12: Different thickness glass measurements

\section{CONCLUSION}

This research work presents a new technique of measuring powder and liquid material's permittivity and permeability, using free space, broadband reflection and propagation techniques. Only initial results are at present available, but the technique shows some promise for future measurement.

\section{ACKNOWLEDGMENT}

The authors would like to acknowledge the support of Bryan Hallissey in the Building Technology Department of Waterford Institute of Technology for their support. 


\section{REFERENCES}

[1] R. M. Redheffer, "The measurement of dielectric constant," Techniques of Microwave Measurements, vol. 2, pp. 591-657, 1966.

[2] H. L. Bassett, "A free-space focused microwave system to determine the complex permittivity of materials to temperature exceeding $2000^{\circ} \mathrm{C}$," The Review of Scientific Instruments, vol. 42, pp. 200-204, 1971.

[3] Yaqiang Liu, "Different definations of Antenna far field boundary," Waterford Institute of Technology, Waterford, 2009.

[4] D.K. Ghodgaonkar, V.V. Varadan and V.K. Varadan, "Free-space measurement of complex permittivity and complex permeability of magnetic materials at microwave frequencies," IEEE Trans. Instrum. Meas., vol. 39, pp. 387-394, April 1990.

[5] W. C. Stone, "Electromagnetic Signal Attenuation in Construction Materials." in NIST Construction Automation Program Report No. 3 , October, 1997.

[6] R. Zoughi, Microwave Non Destructive Testing and Evaluation.Dordrecht,The Netherlands:Kluwer,2000.

[7] Ltd Pentax Precision Co. Introduction Manual Electronic Total Station R-300 Series.[Online]. http://www.geosolution.com/download/r300xquick-basic-english.pdf

[8] Yaqiang Liu ,Paul O'Leary, "Design of Flexible Platform for Various Free-Space, Broadband Dielectric Analysis Measurement Techniques.”, IEEE International Workshop on Electromagnetic,Taipei,Taiwan,2011

[9] Mansor Nakhkash, Yi Huang, Waleed Al-Nuaimy,and M.T.C.Fang “An Improved Calibration Technique for Free-Space Measurement of Complex Permittivity." IEEE Transactions on Geoscience and Remote Sensing, VOL. 39, NO. 2, February 2001 